Journal of Computer Science 7 (9): 1386-1392, 2011

ISSN 1549-3636

(C) 2011 Science Publications

\title{
Medical Image Compression Using Fuzzy C-Means Based Contourlet Transform
}

\author{
${ }^{1}$ M.Tamilarasi and ${ }^{2} \mathrm{~V}$. Palanisamy \\ ${ }^{1}$ Department of Electronics and Communication Engineering, \\ King College of Technology, \\ ${ }^{2}$ Info Institute of Engineering, Tamilnadu, India
}

\begin{abstract}
Problem statement: To meet the demand for high speed transmission of image in efficient image storage and remote medical treatment, the efficient image compression is essential. The contourlet transform along with wavelet theory has great potential in medical image compression. Approach: The significant portion of the medical image applied with Fuzzy C-means based contourlet transform. DWT applied to the rest of the image. Finally modified EZW of six symbols differing from normal EZW was applied to the whole image. This technique increases PSNR and gives better compression ratio. Results: The MATLAB simulation showed that the method of separate transforms to the two regions proves better results compared to the ordinary way of applying only single transforms to the whole image. The results revealed that proposed algorithm was simple and computationally fewer complexes based on embedded block coding with coefficient truncation. Conclusion: The compression of the proposed algorithm is superior to EZW, SPIHT. Our new method of compression algorithm can be used to improve the performance of Compression Ratio (CR) and Peak Signal to Noise Ratio (PSNR). In future this study can be extended to real time applications for video compression in medical images.
\end{abstract}

Key words: Contourlet transform, Peak Signal to Noise Ratio (PSNR), Compression Ratio (CR), Directional Filter Bank (DFB), Region Of Interest (ROI), Laplacian Pyramid (LP), Fuzzy C-means (FCM)

\section{INTRODUCTION}

Past few years have witnessed abundant techniques in image compression, primarily for its widespread usage in internet technologies, medical applications. Video and image applications require intensive data acquisition, storage and processing in order to transmit high quality images through limited bandwidth. Data compression has relieved the burden of image transmission and storage at the cost of extra computationally extensive processing (Olyaei and Genov, 2005). Innovative visualization techniques are therefore needed to assist the radiologist: in approaching the growing amounts of information available to interpret and to perform diagnosis. A large part of the modern medical data is expressed as images or other types of digital signals, such as MRI, CT, PET, Image transformations can be achieved through several transforms like DCT, DFT and wavelet. Contourlet based ROI with wavelet transform of digital signals and images have been a topic of interest for better compression.
Video and image applications require the acquisition of huge amount of such sophisticated image data has give rise to the development of automatic processing and analysis of medical images in order to transmit high quality images through limited bandwidth.

Medical diagnosis becomes effective if it identifies the defective areas in limited processing. In medical images, some structures in the data are of interest. These structures typically occupy a small percentage of the data, but their analysis requires contextual information like locations within a specific organ or adjacency to sensitive structures. Therefore, while focusing on a particular region of the data, designated as a Region Of Interest (ROI), contextual information surrounding that region is important. However, the same amount of detail is not required for the context and the ROI. Fuzzy C-means logic is used to separate out ROI by extracting image features. After performing segmentation contourlet transform is applied to significant region and wavelet transform is applied to rest of the image for better compression ratio.

Corresponding Author: M. Tamilarasi, Department of Electronics and Communication Engineering,

King College of Technology, Tamilnadu, India 
Several algorithms like Shapiro's EZW (Embedded Zerotree Wavelet) (Shapiro, 1993), the SPIHT (Set partitioning in Hierarchical Trees) (Said and Pearlman, 1996) and the SPECK (Set Partitioning Embedded Block) (Islam and Pearlman, 1999) exists for image compression. In (Said and Pearlman, 1993), compression is performed by four symbols different from the one used in the original method to code the wavelet coefficients. The previous works uses four coefficients whereas the proposed technique uses six symbols namely $\mathrm{P}, \mathrm{N}, \mathrm{Z}, \mathrm{T}$, $\mathrm{Pt}$ and $\mathrm{Nt}$ for efficient compression. Huffman coding is then applied to the indices obtained by Modified EZW to get the encoded image.

\section{METERIALS AND METHODS}

In this section, we propose the modification of the EZW algorithm with improved version of contourlet transform for the significant region and DWT for the insignificant region together with fuzzy C-means based segmentation technique. Following this introduction, the remainder of the section is organized as follows: In section II the contourlet transform is explained with some basics of wavelet transform from which it emerges. Our approach of reduction in computational complexity with increased compression ratio using modified EZW algorithm is explained in section III. Section IV deals with the results and finally conclusions are drawn in section $\mathrm{V}$.

Discrete contourlet transform: Wavelet transform is a powerful transform to represent images that contains smooth areas separated with edges, it lacks in its performance when the edges are smooth curves. Researchers have recently come up with a new family of wavelet methods that can capture the intrinsic geometrical structures such as curvelet transform (and Donoho, 2000) and contourlet transform (Do, Martin vetterli, 2005). Contourlets are a sparse efficient expansion for two dimensional signals that are piecewise smooth away from smooth contours (Po and Do, 2003). Curvelets are very successful in detecting image activities along curves, while analyzing images at multiple scales, locations and orientations. The contourlet transform proposed by Do and Vetterli (Do and Vetterli, 2005), uses a structure similar to that of curvelets, except at discrete domain and has good approximation property for smooth $2 \mathrm{D}$ functions and is therefore computationally efficient. It is a multi resolution and directional decomposition of a signal using a combination of Laplacian Pyramid (LP) and a Directional Filter Bank (DFB) Fig. 1a. The LP decomposes images into subbands and DFB analyzes each detail image. Figure $1 \mathrm{~b}$ shows the resulting frequency division, where the whole spectrum is divided both angularly and radially and the number of directions is increased with frequency.

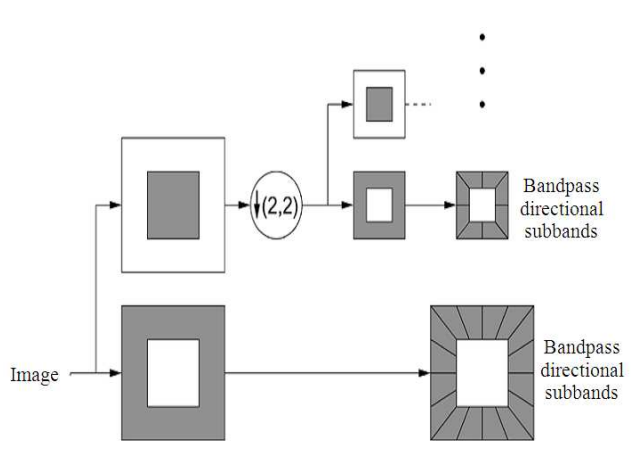

(a)

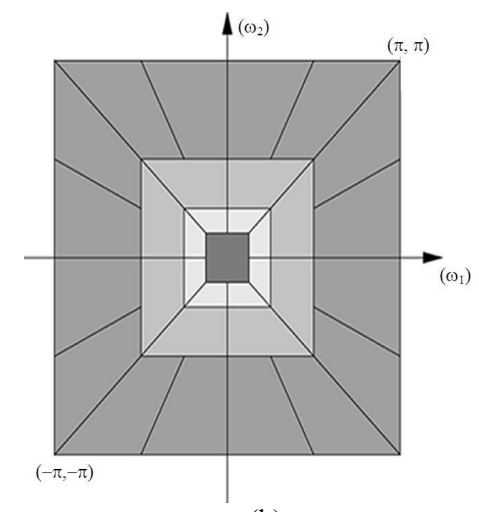

(b)

Fig. 1: The contourlet transform: (a) Block diagram. (b) Resulting frequency division

It was shown that the discrete contourlet transform achieves perfect reconstruction and has a redundancy ratio that is less than 4/3(Duncan and Do, 2006).

Laplacian pyramid: The multiscale decomposition of Directional Filter Bank (DFB) can be achieved by Laplacian Pyramid (LP) which is introduced by Burt and Adelson (1983). The LP decomposition at each level generates a low pass subband and a high pass subband. The low pass sub band is a down sampled coarse version (1/4 size of the original) of the original image and the high pass subband is a detail image (same size of the original) containing the difference between the original and the prediction, resulting in a band pass image (Esakkirajan et al., 2006). The process can be iterated by decomposing the coarse subband repeatedly. If this process is performed continuously, bandpass filtered images corresponding to different bands of frequencies can be obtained, each sampled at successively different densities. A drawback of the LP is the implicit oversampling. However, in contrast with the critically sampled wavelet scheme, the LP has the distinguishing feature that each pyramidal level generates only one bandpass image which does not have scrambled frequencies. 


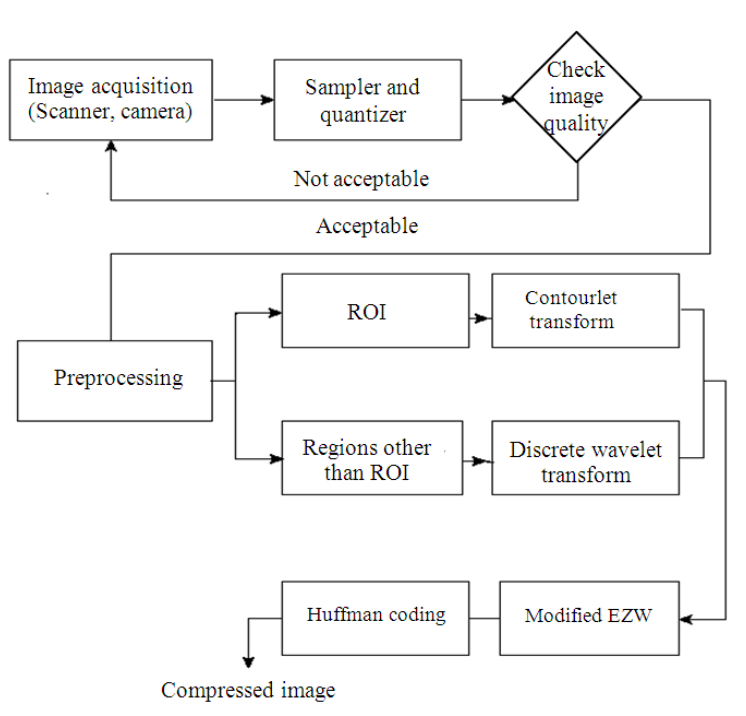

Fig. 2: The Proposed algorithm model

This frequency sampling happens in the wavelet filter bank when a high pass channel after down sampling, is folded back into the low frequency and thus its spectrum is reflected. In LP this effect is avoided by only down sampling the low pass channel.

Directional Filter Bank (DFB): The directional filter bank partitions the frequency plane into a set of wedge shaped band pass regions. An important property of the DFB is its ability to extract 2D directional information of an image, which is important in image analysis. The DFB is efficiently implemented n-level tree structured decomposition that leads to $2^{\mathrm{n}}$ subbands. To obtain the desired frequency partition, an involved tree expanding rule has to be followed. The DFB is designed to capture the high frequency that represents directionality of images and is maximally decimated. This means that the total number of subband coefficients is the same as that of the original image and they can be used to reconstruct the original image without any error.

Proposed scheme: Figure 2 shows the overall system flow diagram. It accepts input image and produces segmented image as output. It consists of various modules namely preprocessing, fuzzy segmentation (Troung et al., 2004). The proposed system starts with the key frame of the medical image, preprocessing of the image is done for removing the noise for a better segmentation. After preprocessing, segmentation and tracking are performed. A model fitting technique is to be proposed after tracking the borders. The tracked borders are to be decomposed into meaningful regional parameters. The original image can be reconstructed from the compressed image using inverse transforms to the above proposed algorithm model.

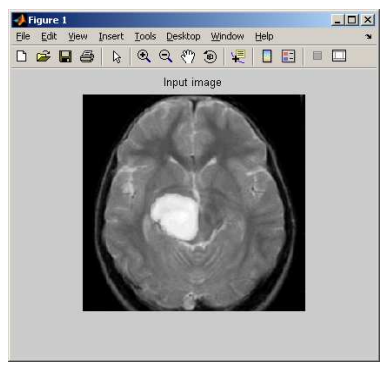

(a)

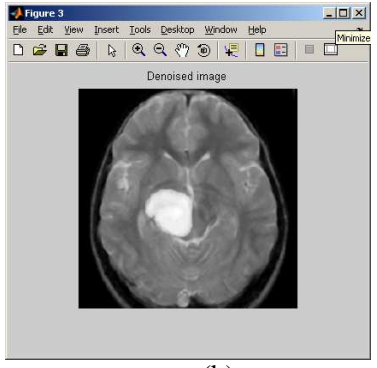

(b)
Fig. 3: Output of the preprocessed image

Noise removal: In order to make the image noise free, preprocessing should be performed as the first step. Preprocessing phase of the images is necessary to improve the quality of the images and make the images more reliable for further processing. In the development of medical image compression usually the tumor region depends on the region of interest, which is usually of low contrast and noisy nature. Hence an image denoising and enhancement may be required to preserve the image quality, highlighting image features and suppressing the noise. In the proposed algorithm, median filter is selected as it removes impulse noise without blurring sharp edges.

The median filter was once the most popular nonlinear filter for removing impulse noise because of its good denoising power and computational efficiency.

The idea of median filtering is simply to replace each pixel value in an image with the median value of its neighbors, including itself. Figure $3 \mathrm{a}$ and $\mathrm{b}$ are the input image and preprocessed images respectively.

Extraction of ROI: The purpose of feature extraction is to reduce the original data set by measuring certain properties, or features, that distinguish one input pattern from another pattern (Anil, 1989). Two textural features namely contrast, correlation based on the Gray Level Cooccurence Matrices (GLCM) have been used in this work.

Spatial gray level co-occurrence estimates image properties relates second order statistics. Haralick (1979) suggested the use of Gray Level Co-Occurrence Matrices (GLCM) which has become one of the well known and widely used texture features. GLCM $\left\{\mathrm{P}_{(\mathrm{d}, \theta)}(\mathrm{i}, \mathrm{j})\right\}$ represents the probability of the occurrence of a pair of gray levels $(i, j)$ separated by a distance $d$ at angle $\theta$. The commonly used unit pixel distances and the angles are $0,45,90$ and $135^{\circ}$. A detailed algorithm of calculation of GLCM $\left\{\mathrm{P}_{(\mathrm{d}, \theta)}(\mathrm{i}, \mathrm{j})\right\}$ has been given in Chen et al. (1998). Textural characteristics like Contrast and Correlation can be captured from images using second order distribution gray levels using the following formulas. 
Contrast:

$S c=\sum_{i} \sum_{j}(i-j)^{2} P(i, j)$

Correlation:

$S_{o}=\frac{\sum_{i j} \sum_{j}(i j) p(i, j)-\mu_{x} \mu_{y}}{\sigma_{x} \sigma_{y}}$

where, $\mu_{\mathrm{x}}, \mu_{\mathrm{y}}, \sigma_{\mathrm{x}}, \sigma_{\mathrm{y}}$ are the means and standard deviations of $\mathrm{p}_{\mathrm{x}}$ and $\mathrm{p}_{\mathrm{y}}$.

Modified Fuzzy C means technique: To separate out ROI from the diagnosis image, textural based fuzzy Cmeans segmentation has performed which plays a dominant role in image analysis. Textural characteristics like correlation, contrast are calculated from the denoised image and fuzzy C-means clustering algorithm is applied to the resultant image. It has a prime contribution in Pattern Recognition (Hemanth et al., 2009).

Clustering is a group of data with similar characteristics. To divide the data into several groups the similarity of objects are used. The distance functions are used to find the similarity of two objects in the data set. Here priori information about classes is not required. Clustering can also be thought of as a form of data compression, where a large number of samples are converted into a small number of representative prototypes or clusters (Negar and Yazdi, 2009).

Fuzzy C-Means (FCM) is a method of clustering which allows one pixel to belong to two or more clusters. The FCM algorithm attempts to partition a finite collection of pixels into a collection of " $\mathrm{C}$ " fuzzy clusters with respect to some given criterion. Some examples of values that can be used as similarity measures include distance, connectivity and intensity. In this work, the images are segmented into four clusters based on the feature values.

Fuzzy C-means algorithm is based on minimization of the following objective function:

$\mathrm{J}\left(\mathrm{U}, \mathrm{cl}_{1}, \mathrm{c}_{2}, \ldots \ldots, \mathrm{c}_{\mathrm{c}}\right)=\sum_{\mathrm{i}=1}^{\mathrm{c}} \mathrm{J}_{\mathrm{i}}=\sum_{\mathrm{i}=1}^{\mathrm{c}} \sum_{\mathrm{j}=1}^{\mathrm{n}} \mathrm{uij}^{\mathrm{m}} \mathrm{dij}^{2}$

$\mathrm{u}_{\mathrm{ij}}=$ Between 0 and 1

$c_{i}=$ The centroid of cluster $i$

$\mathrm{d}_{\mathrm{ij}}=$ The Euclidian distance between $\mathrm{i}^{\text {th }}$ centroid $\left(\mathrm{c}_{\mathrm{i}}\right)$ and $\mathrm{j}^{\text {th }}$ data point

$\mathrm{m}=\in[1, \infty]$ is a weighting exponent
The modified FCM algorithm is based on the concept of data compression where the dimensionality of the input is highly reduced. The data compression includes two steps: quantization and aggregation. The quantization of the feature space is performed by masking the lower ' $m$ ' bits of the feature value. The quantized output will result in the common intensity values for more than one feature vector. In the process of aggregation, feature vectors which share common intensity values are grouped together. A representative feature vector is chosen from each group and they are given as input to modified FCM. Once the clustering is complete, the representative feature vector membership values are distributed identically to all the members of the quantization level. Since this technique uses a reduced dataset, the convergence rate is highly improved.

Algorithm: The entire algorithm can be summarized as follows:

Step 1: Initialize the membership matrix, $\mathrm{U}=\left[\mathrm{u}_{\mathrm{ij}}\right]$. Step 2: At $\mathrm{k}^{\text {th }}$ number of iteration:

Calculate the entire vectors $c_{i}$ with $\mathrm{u}_{\mathrm{ij}}$ :

$c_{i}=\frac{\sum_{j=1}^{n} u_{i j} m_{y_{j}}}{\sum_{j=1}^{n} u_{i j}^{m}}$

Where, $y=$ reduced data set.

Step 3: Update the membership matrix $U$ for the $\mathrm{k}^{\text {th }}$ step and $(\mathrm{k}+1)^{\text {th }}$ step:

$u_{i j}=\frac{1}{\sum_{k=1}^{c}\left(\frac{d_{i j}}{d_{k j}}\right)^{2 /(m-1)}}$

where, $d_{i j}=y_{j}-c_{i}$

Step 4: If $\|\mathrm{U} \quad(\mathrm{k}+1)-\mathrm{U}(\mathrm{k})\|<\quad \varepsilon$ then stop;otherwise return to step 2 .

FCM output with 4 clusters is shown in following Fig. 4.

The ROI segmented output through Fuzzy Cmeans algorithm are subjected to contourlet transform and the region of less significance are performed with Discrete Wavelet Transform. For wavelet comparison we chose 'Haar' wavelets of the first order. The contourlet transform and Haar DWT outputs for the test image are shown in Fig. 5. 


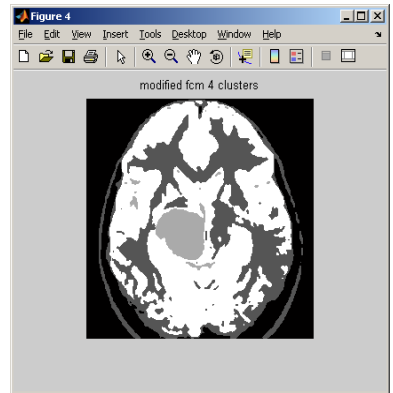

(a)

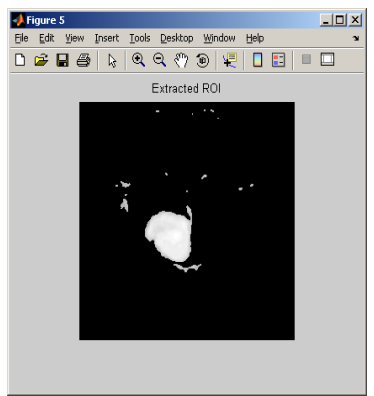

(b)
Fig. 4: Segmented output (a) Fuzzy C-eans(FCM)output (b) ROI output using FCM

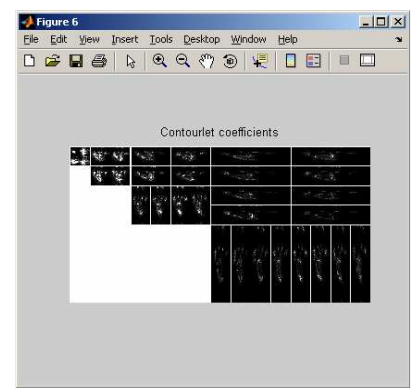

(a)

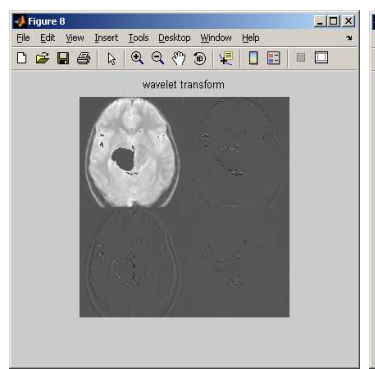

(c)

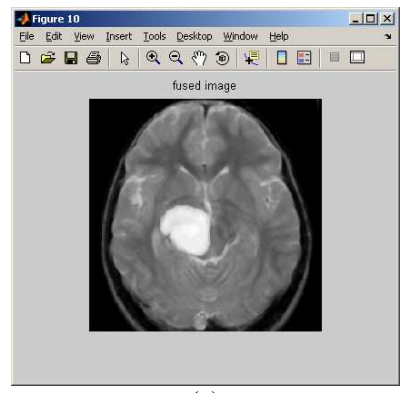

(e)

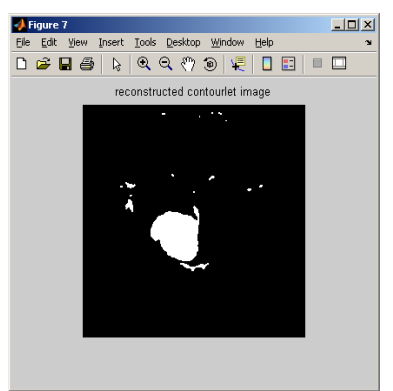

(b)

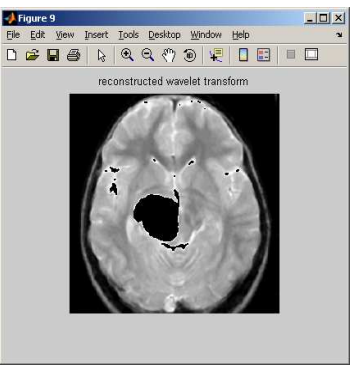

(d) $\begin{array}{cc}\begin{array}{c}\text { (a) Contourlet Coefficients } \\ \text { applied to ROI }\end{array} & \text { (b) Contourlet transform }\end{array}$ output for ROI (c) Wavelet Transform output (d) Reconstructed DWT (e)Fused image combining Contourlet and DWT outputs

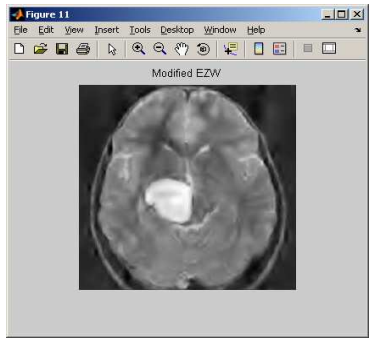

Fig. 6: Modified EZW output

ROI based modified EZW: In Shapiro's EZW algorithm (Esakkirajan et al., 2006), a "zerotree" consist of a parent and its offsprings are insignificant, then the ancestor is coded as zerotree. If the value of the coefficient is lower than the threshold and has one or more significant descendants with respect to 'j'th level, then they are coded as "isolated zero".

The significance symbols of the image coefficients are placed in the dominant list. The amplitudes of the significant coefficients are placed in the subordinate list. Their values in the transformed image are set to zero in order not to undergo the next step. Finally to the above coefficients, Huffman coding is applied.

Modified compression technique: If a coefficient is tested and found to be significant, its offspring's are also tested. If all the coefficients are insignificant, then the descendants are coded with symbols $\mathrm{Pt}$, for positive coefficient and with symbol Nt for negative coefficients. Figure 6 shows the output of the modified EZW.

Huffman coding assigns less codes for coefficients whose probabilities of occurrence is high and vice versa for coefficients whose probabilities of occurrence is low. The significant size is obtained by binary regrouping of several symbols.

Further all the possibilities regarding the coefficient are to be worked with and have to perform for the different iteration levels. Further the proposed method proves to yield better result with limited computational complexity.

This error can be overcome by Huffman coding in the proposed coding which yields better results with computationally efficient technique.

\section{RESULTS AND DISCUSSION}

The method of separate transforms to the two regions proves better results compared to the ordinary way of applying only single transforms to the whole image. The proposed technique of modified EZW for a 8 -bit $256 \times 256$ images were tested. In the proposed method of compression, to take the whole value as array of bytes, the medical image values having similar attributes are coded and taken as a sequence of bits. 


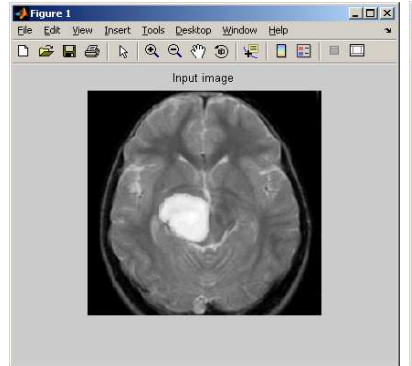

(a)

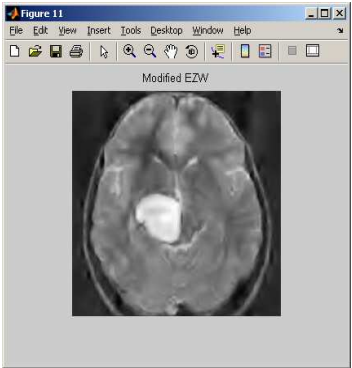

(b)
Fig. 7: (a) Input image (b) Reconstructed image

\begin{tabular}{|c|c|c|c|c|c|c|}
\hline \multirow[b]{2}{*}{ Type } & \multicolumn{2}{|l|}{ EZW } & \multicolumn{2}{|l|}{ SPIHT } & \multicolumn{2}{|c|}{ MODIFIED EZW } \\
\hline & PSNR & $\mathrm{CR}$ & PSNR & $\mathrm{CR}$ & PSNR & $\mathrm{CR}$ \\
\hline est & 34.16 & $8: 1$ & 36.28 & $16: 1$ & 32.08 & $17: 1$ \\
\hline Test MRI image2 & 32.04 & $20: 1$ & 33.16 & $30: 1$ & 31.84 & $31: 1$ \\
\hline
\end{tabular}

The Region of Interest region is coded using the contourlet transform and the remaining portions are coded using Haar Wavelet filter. Figure $7 \mathrm{a}$ and $\mathrm{b}$ shows the input and reconstructed Images.

The compression ratio for contourlet based modified EZW increases than the normal EZW algorithm. The PSNR and CR for the proposed algorithm is shown in Table 1

\section{CONCLUSION}

In this section, we proposed a new image transform called MEZW to compress medical image based on the combination of the wavelet transform and the nonsampled directional filter banks. The proposed algorithm is simple and computationally less complex which is based on embedded block coding with coefficient truncation. Further addition of two new symbols results in efficient compression with reduced computational time. The compression of the proposed algorithm is superior to EZW, SPIHT, Our new method of compression algorithm can be used to improve the performance of Compression Ratio (CR) and Peak Signal to Noise Ratio (PSNR). In future this work can be extended to real time applications for video compression in medical images. The result shown above reveal the superior performance of contourlet against wavelet transform at higher compression ratios. However at lower compression ratios wavelet transform proves a suitable approach.

Formulas: The bit per pixel (bpp) and PSNR for the arbitrary shaped region is evaluated by the following. The PSNR is the measure of quality of reconstruction of lossy compression codecs:

$$
\text { PSNR }=10 \log \frac{\text { MAX }^{2}}{\frac{1}{w \times h} \sum_{i=1}^{w} \sum_{j=1}^{h}(o(i, j)-c(i, j))^{2}}
$$

Where:

Oij $=$ The original image

$\mathrm{Cij}=$ The reconstructed image

$\mathrm{w}=$ The total number of row elements

$\mathrm{h}=$ The total number of column elements

MAX $=255$ :

$\mathrm{CR}(\mathrm{bpp})=\frac{\text { number of coded bits }}{\mathrm{n} \times \mathrm{m}}$

where, $\mathrm{n}, \mathrm{m}$ is the image size.

\section{REFERENCES}

Anil, K.J., 1989. Fundamentals of Digital Image Processing. 1st Edn., Prentice Hall publications, Englewood Cliffs, NJ, ISBN: 0133361659, 9780133361650, pp: 569.

Burt, P.J. and E.H. Adelson, 1983. The Laplacian Pyramid as a Compact Image Code. IEEE Trans. Commun., $\quad 31$ : 532-540. DOI: 10.1109/TCOM.1983.1095851

Candes, E.J. and D.L. Donoho, 2000. Curvelets-a surprisingly Effective Nonadaptive Representation for Objects with Edges. In: Curve and Surface Fitting, Cohen, A., C. Rabut and L.L. Schumaker (Eds.). Vanderbilt University Press, Saint-Malo, pp: 105-105 ISBN0826513573

Chen, H., L.F. Pau and P.S.P. Wang, 1999. The Handbook of Pattern Recognition and Computer Vision. 2 Edn., World Scientific, Singapore, pp: 1019. ISBN9810230710, 9789810230715

Do, M.N. and M. Vetterli, 2005. The contourlet transform: An efficient directional multiresolution image representation. IEEE Trans. Image Proc., 14: 2091-2106. DOI: 10.1109/TIP.2005.859376

Esakkirajan, S., T. Veerakumar, V. Senthilmurugan and R. Sudhakar, 2006. Image compression using contourlet transform and multistage vector quantization. GVIP J., 6: 19-28. Haralick, R.M., 1979. Statistical and structural approaches to texture. Proc. IEEE, pp: 67: 786-804. DOI: 10.1109/PROC.1979.11328

Hemanth, D.J., D. Selvathi and J. Anitha, 2009. Effective fuzzy clustering algorithm for abnormal MR brain image segmentation. Proc. ISCC., 1: 609-614. DOI: 10.1109/IADCC.2009.4809081 
Islam, A. and W.A. Pearlman, 1999. An embedded and efficient low-complexity hierarchical image coder. In: visual communication image process. Proc. SPIE, 3653: 294-305. DOI: 10.1117/12.334677

Olyaei, A. and R. Genov, 2005. Mixed-Signal Haar Wavelet compression Image Architecture. Proceeding of the 48th Midwest Symposium on Circuits and Systems, Aug. 7-10, IEEE Xplore Press, Covington, KY, pp: 1267-1270. DOI: 10.1109/MWSCAS.2005.1594339

Po, D.D.Y. and Do, 2003. Directional multiscale statistical modeling of images. Proceeding of the SPIE Conference on Wavelet Applications in Signal and Image Processing X, August 04, SPIE Digital Liberary, San Diego, USA, pp: 69-79. DOI: 10.1117/12.506412

Po, D.D.-Y. and M.N. Do, 2006. Directional multiscale Modeling of Images using the Contourlet Transform. IEEE Trans. Image Proces., 15: 16101620. DOI: $10.1109 /$ TIP.2006.873450
Said, A. and W.A. Pearlman, 1996. A new fast and efficient image codec based on set partitioning in hierarchical trees. IEEE Trans. Circuits Syst. Video Technol., 6: 243-250. DOI: 10.1109/76.499834

Said, A. and W.A. Pesrlman, 1993. Image compression using the spatial-orientation tree. Proceeding of the IEEE Int. symposium on Circuits and Systems, May 3-6, IEEE Xplore Press, USA, pp: 279-282.

Shapiro, J.M., 1993. Embedded image coding using zerotrees of wavelet coefficients. IEEE Trans Signal Process, 41: 3445-3462. DOI: 10.1109/78.258085

Troung, T.N. and S. Oraintara, 2004. Textural classification using non-uniform directional filter bank. Proceeding of the IEEE 3rd Digital Signal Processing Workshop and IEEE Signal Processing Education Workshop, Aug. 1-4, IEEE Xplore Press, USA, pp: 147-150. DOI: 10.1109/DSPWS.2004.1437930 